\section{Kommentare und Hinweise}

O. Colhoun

Institut für Laboratoriumsmedizin, Klinikum Frankfurt

Höchst, Frankfurt am Main, Deutschland

Englischer Begriff comments and hints

Definition Kurze Erläuterungen $\mathrm{zu}$ den Messwerten (s. \ Messwert) im Befund der $\triangleright$ Labor-EDV.
Beschreibung Sie sind Teil des Messwerts, da sie zur medizinischen Bewertung desselben herangezogen werden müssen (Hinweis auf Hämolyse, v. a. Patientenverwechslung etc.). Kommentare sollen daher stets eindeutig dem Messwert ( $\triangleright$ Messwertprotokoll) zugeordnet werden und auf demselben Befundblatt wie dieser abgedruckt sein. Die Charakterisierung bestimmter Parameterkonstellationen markiert den Grenzübergang zum $>$ Befund, hinterlegte Regelwerke und Berechnungen für die automatische Generierung von Kommentaren aufgrund von Messwerten den Übergang zu wissensbasierten Expertensystemen (s. - Expertensystem). 